\title{
Perfil psicossocial de indivíduos com disfunção temporomandibular (DTM) atendidos no projeto de extensão alívio
}

\author{
Natália Wandekoeken Silvestre (iD, Carlos Henrique Cardoso Sarcinelli iD, Ana Giulia Prado Cazelli (D), \\ Cintia Helena Santuzzi ${ }^{D}$, Dhandara Araújo De Souza ${ }^{\mathbb{D}}$, Fernanda Mayrink Gonçalves Liberato ${ }^{(D)}$
}

Universidade Federal do Espírito Santo, Vitória, Espirito Santo, Brasil

\section{Introdução}

As disfunções temporomandibulares (DTM) são condições que frequentemente evolvem para cronicidade e o perfil psicossocial do indivíduo pode contribuir para o seu prognóstico.

\section{Objetivos}

Traçar o perfil psicossocial de pacientes com DTM atendidos no Projeto Multiprofissional de Dor Orofacial da Universidade Federal do Espírito Santo (Alívio).

\section{Material e métodos}

Trata-se de um estudo descritivo retrospectivo, aprovado no Comitê de Ética em Pesquisa (CAAE: 48433621.1.0000.5060). Foram analisados dados dos pacientes no período de novembro de 2020 a agosto de 2021.

Para análise do perfil psicossocial tivemos como base: Índice de Qualidade do sono de Pittsburgh (PSQl); Cinesiofobia (TAMPA); Hábitos orais (OBC); Hipervigilância (PVAQ); Catastrofização (PCS), a prática e o tipo de atividade física realizada. Os resultados foram analisados através de média, desvio padrão e frequência.

\section{Resultados \\ Compuseram a amostra 18 indivíduos dos quais 66,7\% apresentaram distúrbio do sono (PSQI); 66,7\% apresen- tavam baixa cinesiofobia (TAMPA), 83,3\% demonstraram hábitos orais que representam alto risco em desenvolver DTM (OBC); hipervigilância (PVAQ) com média de pontuação 56,78 (16,3) de um total de 80 pontos; 38,9\% foram classificados com baixo nível catastrofização (PCS); 55,6\% praticavam atividade física, destes, 33,6\% tinham como hábito a prática de caminhada. A análise psicossocial de pacientes com DTM considera aspectos psicológicos e sociais no início e prognóstico desse transtorno. Logo, faz-se necessária uma abordagem psicossocial e multidisciplinar para entender e abordar fatores além dos físicos que repercutem no prognóstico da condição.}

\section{Conclusão}

O presente estudo observou que existe uma relação que pacientes com DTM dispõe de uma predominância de distúrbio do sono, baixa cinesiofobia, hábitos orais relacionados a alto risco de desenvolver DTM, hipervigilância com pontuação média, baixo nível de catastrofização e a prática de atividade física é moderada.

Palavras-chave: Disfunção temporomandibular, Biopsicossocial, Multiprofissional 